\title{
EL PROFESOR UNIVERSITARIO DE AMÉRICA LATINA: HACIA UNA RESPONSABILIDAD ÉTICA, CIENTÍFICA y SOCIAL
}

\author{
Dra. Diana Soto Arango ${ }^{1}$ \\ Universidad Pedagógica y Tecnológica de Colombia \\ Grupo de Investigación HISULA \\ RUDECOLOMBIA \\ dianaelvirasoto@gmail.com
}

Recepción: 07/10/2009

Evaluación: $15 / 10 / 2009$

Aceptación: 19/10/2009

Artículo de Reflexión

\section{RESUMEN}

El trabajo tiene como objetivo analizar la responsabilidad ética-científico-social del profesor universitario en América Latina desde su ejercicio docente en una institución como es la universidad que presenta cambios estructurales en el siglo XXI. Se aborda la investigación desde la pregunta ¿si la universidad en América Latina ha formado su cuerpo docente con capacidad profesional de liderar la transformación culturaleducativa, y de liderar los cambios científicos-tecnológicos-sociales desde la ética, $l a$ solidaridad, la tolerancia, la inclusión, en las "sociedades del saber" cambiantes del siglo XXI. La metodología se centra en la historia social de la educación donde el docente se analiza dentro del contexto socio-político de las universidades latinoamericanas. Las fuentes primarias de Leyes, Decretos, informes sustentan el trabajo. Se localizó, en el estudio, que se requiere una redefinición conceptual y legal de la práctica y rol del profesor universitario dentro de los criterios de la nueva visión de la universidad Latinoamericana. Se concluye que el profesor universitario debe demostrar el conocimiento de la disciplina y la didáctica específica unido a las redes de

\footnotetext{
${ }^{1}$ Profesora Titular de la Universidad Pedagógica y Tecnológica de Colombia. Organizó y fue la primera Directora del Doctorado "Ciencias de la Educación" de RUDECOLOMBIA. Directora de Grupo de Investigación «Historia y Prospectiva de la Universidad Latinoamericana» - HISULA, desde la fundación en 1992.
} 
investigación internacionales, pero encontramos también, que son indispensable los valores personales de solidaridad y cooperación frente a la sociedad de la individualidad y la falta de tolerancia. Se recomienda nuevos estudios que respondan si jestán nuestras universidades y docentes preparados para el cambio conceptual de la universidad dentro de las sociedades del saber del siglo XXI

Palabras clave: Profesor, Catedrático, Universidad, América Latina, Ética.

\title{
THE LATIN AMERICAN UNIVERSITY PROFESSOR: TOWARDS AN ETHICAL, SCIENTIFIC AND SOCIAL RESPOSIBILITY
}

\author{
Dra. Diana Soto Arango \\ Universidad Pedagógica y Tecnológica de Colombia. \\ RUDECOLOMBIA \\ HISULA Research Group \\ dianaelvirasoto@gmail.com
}

\begin{abstract}
The topic of " ethical- Scientific- social responsibility" of a university professor in Latin America is introduced from the question whether the Latin America university has trained and is actually training its teaching faculty with professional capacity to lead the educational and cultural transformation, the social, technological and scientific changes, all of them seen from ethics, solidarity, tolerance, and inclusion in the everchanging "knowledge societies" of the 21th century. Both mission and vision of the 21 th century Latin America university are co-related to the university professor's profile.
\end{abstract}

Key words: professor, university, Latin America, ethics

\section{INTRODUCCIÓN}

En este trabajo se analiza el tema del "El profesor universitario de América Latina. Responsabilidad científico-social”. Sobre los dos ejes que se investigan: profesor y la universidad. Hay que decir, que, se cuentan por separado con obras clásicas pero paradójicamente la temática de la formación en la "responsabilidad ética-científicosocial" del profesor universitario se ha ignorado y sólo recientemente se cuentan con algunas reflexiones sobre este tema crucial que indicamos en este estudio. 
El marco general de nuestro estudio se da a través del problema que indicamos con la pregunta ¿si la universidad en América Latina ha formado y está formando su cuerpo docente con capacidad profesional de liderar la transformación cultural-educativa, y de liderar los cambios científicos-tecnológicos-sociales desde la ética, la solidaridad, la tolerancia, la inclusión en las "sociedades del saber" cambiante del siglo XXI.

Abordaremos primeramente, el tema de la conceptualización de la universidad desde el marco histórico y de la prospectiva del siglo XXI con su misión y visión para luego, en una segunda parte, centrarnos en el estudio especifico de la formación del docente universitario. Finalmente, unas conclusiones reflexivas desde la realidad del docente universitario ${ }^{2}$ en Latinoamérica de cómo se llega a ser profesor universitario y cómo actúa sobre las personas que forma.

\section{La universidad latinoamericana versus formadora de docentes}

El profesor universitario se encuentra inmerso en una institución y una sociedad. Por lo tanto, es importante reconocer la historia de las instituciones universitarias ${ }^{3}$ y de la formación de los docentes dentro de su contexto socio-político local, nacional e internacional. De esta manera, se entenderá las nuevas demandas sociales hacia una institución que se mira como la formadora del personal que dirige esa sociedad ${ }^{4} \mathrm{y}$ con un modelo educativo que se cuestiona al no cumplir con la prospectiva de la nueva sociedad globalizante, del espacio común del conocimiento y en especial con las "sociedades del saber" que permiten la dimensión de la transformación cultural, sociopolítica-económica e institucional ${ }^{5}$ como lo destaca la UNESCO.

En el siglo XVIII la Universidad era la institución que otorgaba los grados académicos. La Universidad pública se concebía como: la institución que estaría

\footnotetext{
2 Para este trabajo asumimos conceptualmente que el profesor o docente universitario es aquel que realiza actividades de docencia vinculadas con la investigación y proyección a la comunidad.

3 Destacamos como primera universidad "oficial" dependiente del Rey, fue la de Nápoles (1224), fundada por Emperador Federico II y la de Palencia (1220) en España. Por su parte, el Papa Gregorio inicio las universidades Pontificias con la de Toulouse (1224). Hemos localizado que la primera que se crea por iniciativa privada es la de Gotinga (1734) por parte del Barón de Munchhausen. Igualmente, la primera legislación universitaria aceptada por la corporación se estableció con Alfonso X el Sabio (1221-1284). SOTO ARANGO, Diana. (2007): La autonomía en la universidad colombiana. Nuevo Reino de Granada, en Autonomía y Modelos Universitarios en América Latina. SOTO ARANGO, Diana, LAFUENTE GUANTES, María Isabel, Tomo II Colección Historia y Prospectiva de la Universidad Latinoamericana. Universidad de León, España, Asociación Colombiana de Universidades, ASCUN, Universidad Pedagógica y Tecnológica de Colombia, RUDECOLOMBIA.

4 El concepto de universidad no ha sufrido mayores cambios del esbozado por Pablo de Olavide en 1768, por que continúa en América Latina siendo profesionalizante y no investigadora.

5 SOTO ARANGO Diana. (2009): "Los doctorados en Colombia. Un camino hacia la transformación universitaria", en Revista Historia de la Educación Latinoamericana, número 12, Tunja, Universidad Pedagógica y Tecnológica de Colombia, RUDECOLOMBIA, Sociedad de Historia de la Educación Latinoamericana, pp. 169-170.
} 
bajo la normativa legal del Estado, financiada por éste y tendría por finalidad la formación del sector del gobierno. ${ }^{6}$ En este caso, nos referimos al contexto de las reformas borbónicas que se inscribe, lógicamente, dentro de la variante hispánica ilustrada, ${ }^{7}$ y corresponde al auge del reformismo borbónico a partir de la segunda mitad del siglo XVIII en el cual la universidad se consideró pilar para las reformas ${ }^{8}$ de los cambios socio-económicos-culturales.

En esta época a los profesores de la universidad se les denomina catedráticos ${ }^{9} \mathrm{y}$ eran formados por la misma institución. De hecho, para ser catedrático debía presentar su grado universitario. Las universidades Mayores de América colonial que ostentaban la Cédula Real, se fundamentaban las Constituciones en las de la Universidad de Salamanca. En este sentido, "el catedrático representaba a los miembros más importantes entre las autoridades académicas, encargados de la función primordial de la institución: ejercer la docencia en nombre de la universidad." ${ }^{10}$ La selección del catedrático se efectuaba mediante concurso ${ }^{11}$ en las Universidades Mayores que eran las que estaban aprobadas por Cédula Real y algunas, a su vez, tenían Bula del Papa. Por el contrario, en las Universidades Menores, de las comunidades religiosas, el nombramiento de los citados catedráticos era realizado por el General de la Comunidad.

Hay que decir, que no existía como tal una formación de catedrático. En tal sentido, era una opción del egresado de la universidad optar a los concursos para dedicarse a la docencia. En América colonial, el abogado o el médico tenían la opción de quedarse como catedrático de la universidad hasta que consiguiera otro empleo en la administración del gobierno. Algunos, intercalaban el trabajo docente con el de la profesión. Sin embargo, para el Fiscal Moreno y Escandón, principal reformador de los estudios superiores en Nueva Granada, era prioritario el tener la financiación de las cátedras para que de esta manera los catedráticos "sin divertirse a otro objeto,

6 SOTO ARANGO, Diana. (2003): El Modelo de Reforma Universitaria Ilustrada en la Metrópoli y Colonias Americanas, Córdoba, Universidad de Córdoba.

7 Véase la colección "La Ilustración en América colonial", coordinada por SOTO ARANGO, Diana SOTO ARANGO, Diana y PUIG-SAMPER, Miguel Ángel desde el año 1996.

8 SOTO ARANGO, Diana. (2004): La reforma del Plan de Estudios del Fiscal Moreno y Escandón. 1774-1779, Bogotá, Universidad Colegio Mayor del Rosario.

9 Al docente en la universidad se le denominaba catedrático. La cátedra era el "Conjunto de enseñanzas que impartían un saber específico y estaban compuestas por cursos que comprendían una enseñanza específica con una duración de un año académico", en SOTO ARANGO, Diana. (2004): p. 166.

10 "La Cátedra era la piedra fundamental del edificio universitario, símbolo de la transmisión del saber. A ella se ascendía por el mérito debidamente probado en las oposiciones". RODRIGUEZ CRUZ, Águeda. (1990): Historia de la universidad de Salamanca. Salamaca, Fundación Ramón Areces, pp.167-168.

11 En Santa Fe se logra a partir de la Reforma de Moreno y Escalón, véase en SOTO ARANGO, Diana. (1993): Polémicas universitarias en Santa Fe de Bogotá. Siglo XVIII. Bogotá, Universidad Pedagógica Nacional. COLCIENCIAS, pp. 168-169. 
acudan a llenar las obligaciones de su ministerio."12 Es más, se solicitaba que el profesor se alojará en el claustro universitario. ${ }^{13}$

Adquiere importancia el hecho que en el siglo XVIII se inicia una gran campaña contra la enseñanza escolástica. En Nueva Granada como hemos indicado en otros estudios, a partir de Moreno y Escandón, Eloy Valenzuela, entre otros, se impulsa una nueva concepción en la enseñanza partiendo de la experimentación. ${ }^{14}$ En cualquier caso, el catedrático universitario de la época colonial era modelo a seguir por sus estudiantes.

Hay que tener en cuenta que en América colonial española al finalizar el siglo XVII sólo se tenían como públicas las Universidades de México y Lima. En el siglo XVIII con la expulsión de los jesuitas en 1767 se hizo necesario crear nuevas universidades por el vacío que habían dejado las instituciones de la comunidad de San Ignacio. Con respecto, al virreinato de la Nueva Granada se inicia, a partir de esa fecha, una gran polémica del sector civil ${ }^{15}$ por secularizar los estudios superiores. Situación a la que se opuso principalmente la comunidad de Santo Domingo.

En el siglo XIX la universidad hispánica se debatió en buscar una identidad en cada nueva República. En efecto, la educación se considero uno de los pilares fundamentales en la conformación de las nacientes repúblicas. Se le dio relevancia a la educación primaria y a la formación del docente para esta educación. Es en este sentido cuando los nuevos gobernantes asumen la organización de las primeras Escuelas Normales ${ }^{16}$ para formar los docentes de la primaria y secundaria. De esta manera, las

${ }^{12}$ MORENO Y ESCANDON, Francisco Antonio. (1774): Método provisional e interino de los estudios que han de observar los colegios de Santafé por ahora, y hasta tanto se erija Universidad Pública, o su Majestad dispone otra cosa, Santafé, septiembre 22 de 1774, en Archivo General de Indias. Sección Quinta, Audiencia de Santa Fe, Legajo 759.

13 SOTO ARANGO, Diana. (1993): Polémicas Universitarias en Santa Fe de Bogotá Siglo XVIII, Bogotá, Universidad Pedagógica Nacional - COLCIENCIAS.

${ }^{14}$ Por ejemplo para la enseñanza de la Botánica indicaba que "Se propondrán algunas descripciones en las que y en el huerto de la casa, como también en el campo en los domingos de rusticación, se les hará ver prácticamente cuanto se les había enseñado en la clase. Se concluirá este tratado con índice o catálogo de las plantas exóticas que más ocurren en el comercio como lino, cáñamo, canela, clavo, malaguera, sen, ruibarbo, etc., definidas y especificadas según los principios del ya citado Linne", véase en VALENZUELA, Eloy. (1806): Plan que ha de servir de método para el estudio de la filosofia en el Colegio de San Pedro Apóstol de la Villa de Mompox. Biblioteca Nacional de Colombia. Sala Raros y Curiosos, Sección Manuscritos, No. 358, folios 298 a 309.

${ }^{15}$ Un estudio sobre esta polémica por la secularización se localiza en SOTO ARANGO, Diana. (1993): Polémicas universitarias en Santa Fe de Bogotá. Siglo XVIII. Doc. Cit.

${ }^{16}$ Las primeras Escuelas Normales, se crearon en Colombia, Perú y México en el año 1822. En el caso colombiano, La ley del 26 de enero de 1822 cristalizó la apertura de la primera Escuela Normal en Bogotá." VALENCIA, Carlos Hernando. (2006): Las Escuelas Normales $y$ la formación del magisterio. Primera mitad del siglo XX. Manizales, RUDECOLOMBIA, Universidad de Caldas, pp. 42-43. 
Escuelas Normales se originaron bajo el amparo estatal con la influencia francesa, ${ }^{17}$ alemana $^{18}$ y las corrientes pedagógicas que circulaban en la época desde Lancaster, Locke, Pestalozzi, Herbart, entre otros. Es en este contexto que a finales del siglo XIX se crean los Institutos pedagógicos ${ }^{19}$ algunos de los cuales dan origen a las Universidades Pedagógicas en el Siglo XX. ${ }^{20}$ En el caso colombiano se crea mediante el Decreto 547 del 23 de febrero de 1954 denominándola "Universidad Pedagógica femenina."21

No obstante, la formación del profesor universitario en general no presentó cambios y se continuo realizando en la universidad con el mismo criterio de la colonia de ser un primer eslabón en su vida profesional o utilizando parte de su tiempo en la docencia y en el ejercicio de su profesión.

El siglo XX lo iniciamos con un gran movimiento estudiantil que tenía como objetivo repensar la universidad. Pero debemos indicar que sólo hasta finales de siglo XX se dio una corriente de Reformas universitarias en América Latina tratando de ingresar a esta institución en una tardía modernidad. La formación de los docentes que se van a dedicar a la enseñanza en los tres niveles: primaria, secundaria y universitaria se establece, a finales de siglo como una corriente generalizada, que deben obtener el grado en la Universidad. Por lo tanto, las transformación de las Escuelas Normales se convierte en una necesidad relevante que aún siguen funcionando entre un paso de ingreso a las Facultades de Educación. ${ }^{22}$ Este siglo XX, por los cambios científico-

\footnotetext{
17 "El ministro francés Lakanal es quien emplea, en primera instancia, en su informe del 30 de octubre de 1794 el concepto asignado a la Escuela Normal de París, denominándola Escuela Normal porque era la Escuela que daba la norma docente. Ibídem, p.24.

${ }^{18}$ Los orígenes de las Escuelas Normales, como centros educativos, se remontan a los seminarios escolásticos para la preparación de profesores, bajo la influencia del movimiento didactizante impulsado por "Ratke y Comenio" En Austria hacia 1780 existían 15 Escuelas Normales. Ibídem., pp. 23-27

19 SALGADO PEÑA, Ramón Ulises. (2006): "La formación docente en la región: de las normales a las universidades", en Informe sobre la educación superior e América Latina y el Caribe 2000-2005. Caracas, Instituto Internacional de la UNESCO para la Educación Superior de América Latina y el Caribe, p. 174

${ }^{20}$ En Colombia se crea el Instituto Pedagógico Nacional IPN , en el año 1927, en Bogotá, bajo la dirección de la alemana Franzisca Radke que luego en 1954 pasa a denominarse Universidad Pedagógica Nacional.

${ }^{21}$ La directora la Dra. Francisca Racke abogo porque fuera solo de mujeres aduciendo que "las señoritas no va a educar muchachos". ACTA, Consejo Directivo Universidad Pedagógica y Tecnológica de Colombia. UPTC., numero 7 de 1954. Archivo Histórico. UPTC, Véase un análisis de esta situación en HAMON, Ana. (2009): Las mujeres docentes en la Universidad Pedagógica y Tecnológica de Colombia. UPTC. 1961/1980. Tesis doctoral. Doctorado Ciencias de la Educación DE LA Universidad Pedagógica y Tecnológica de Colombia. UPTC., RUDECOLOMBIA.

${ }^{22}$ En Colombia se crean las Facultades de Educación adscritas a la Universidad Nacional en el año 1933. En 1936 mediante la Ley 36 se organiza "la Escuela Normal Superior Universitaria". Comenta Francisco Socarrás que "La Facultad de educación se inició en 1933 con el curso de Información, bajo la rectoría de Rafael Bernal Jiménez" SOCARRÁS, José Francisco (1987): Facultad de Educación y Escuela Normal Superior. Su historia y aporte científico, humanistico y educativo. Tunja, Ediciones la Rana y el Águila, pp. 24-25.
} 
tecnológicos, hace que la universidad sea replanteada y sus docentes requieran de una cualificación para la enseñanza desde las herramientas didácticas para aquellos que ejercen la docencia en la universidad.

Cabe preguntarse, en este contexto, ¿qué incidencia ha presentado, para algunos países de América Latina, el hecho que la universidad otorgué el grado a los docentes de los niveles de educación básica primaria y secundaria?, y ¿Qué se exija mayor cualificación al profesor universitario? ¿Cómo se asume la expansión universitaria y su repercusión en los profesores universitarios? Resulta interesante que la respuesta se dio de inmediato. En el primer caso, en el aumento de las Facultades de Educación en las universidades privadas. Por ejemplo, en Colombia, en el año 2004 sólo 25 Facultades de educación eran públicas y 45 privadas. ${ }^{23}$ Para algunos países este fue un factor agregado en la tendencia de futuro, como lo muestran las estadísticas, de privatizar los estudios superiores. ${ }^{24}$

En el segundo caso, adquiere importancia el hecho que en América Latina se presentó el "bom" de los postgrados. Esta situación con llevó, asimismo al aumento de la matricula y de las instituciones privadas en el nivel universitario. ${ }^{25} \mathrm{Al}$ iniciar el nuevo milenio, por ejemplo, la matrícula de los postgrados en Colombia había aumentado en el $400 \%$ llegando a 55.911 estudiantes. Se concentraba la matrícula en las especializaciones con 49.773 estudiantes; 5.793 en Maestría; 345 en doctorado. La proporción mayoritaria de la matrícula se concentraba en la universidad privada y específicamente en las especializaciones al pasar de 5.214 en 1990 a 38.417 en el año $2000 .{ }^{26}$ Lo anterior es explicable, dado que las especializaciones no requerían de investigación y eran necesarias para el escalafón docente de los profesores.

Pero si tomamos el análisis desde el año 1990, encontramos en el estudio del CNA, para Colombia, que en el año 2008 existía una matrícula de 1.946 doctorandos bajo la tutoría de 1.748 profesores adscritos a ellos con 92 programas. ${ }^{27}$ Es relevante

\footnotetext{
23 "Las Facultades de Educación son unidades para la formación de pregrado y posgrado y hacen parte del sistema nacional de Formación de Educadores. Son 70 en el país". CALVO, Gloria. (Coordinadora) (2004): La formación de los docentes en Colombia. Bogotá, ASCUN, UNESCO, IESALC, p.81.

${ }^{24}$ En Brasil y Colombia, en 1950 el 70\% de las Instituciones de Educación Superior eran de carácter oficial. Esta proporción bajo en el año 1995, al 46\% y en las universidades se redujo al $40 \%$.

${ }^{25}$ En América Latina se paso del año 1994 con el 17,6\% al año 2003 con el 20,5\%. Fuente. IESAL. La tasa bruta corresponde a la relación de la matrícula sobre población de 20 a 24 años. Este dato no es significativo dado que no cubre la relación real de la matrícula. Citado por RAMA, Claudio. (2006): p. 21.

${ }^{26}$ Fuente: SNIES, a febrero de 2009. Observatorio de la universidad colombiana. En el año 2000 se presentaba en matricula de especializaciones: 38.417 en la Universidad privada y 11.356 en la oficial: En la Maestría: 3.255 en la Universidad privada y 2.538 en la oficial. En el doctorado: 266 en la Universidad Oficial y 79 en la privada.

${ }^{27}$ CNA. (abril, 2008): p.3. Según el Ministerio de Educación Nacional de Colombia, aumento a 1.650 estudiantes con 62 programas que presentaban el Registro calificado en 22 universidades colombianas. OBSERVATORIO DE LA UNIVERSIDAD. Febrero de 2009.www.universidad.edu.co. Fuente: SNIES, a febrero de 2009.
} 
indicar que en al año 2002 en América Latina el 6\% de los profesores universitarios tenían formación doctoral mientras en el Reino Unido presentaba un 44\%. A este respecto. Sin embargo, la tendencia de futuro, en América Latina es el de impulsar la formación de doctores y cualificar el personal docente de las universidades. Pero se reconoce que los desarrollos son desiguales en la formación de doctores. ${ }^{28}$ Respecto a los graduados en el nivel de doctorado la diferencia es abismal si nos relacionamos con países como Estados Unidos que en el año 2005 otorgo el título de doctor a 52.855; Brasil a 8.887; México a 1783 y en Colombia en ese año se graduaron $63 .{ }^{29}$ Hay que decir que en el año 2007 se aumento el número de graduados a $102^{30}$ cuando Brasil, en el 2008, gradúo a 11.000 doctores. ${ }^{31}$ En Colombia hasta el 2008 se habían graduado en total 584 doctores. $^{32}$ De estos, 88 se graduaron antes del 2000, los otros 496 se graduaron entre el año 2000 yel $2007 .{ }^{33}$ En definitiva, en Colombia sólo llegaban a 2.422 profesores con este título académico de doctor en los Instituciones de Educación Superior para un porcentaje del 3,1\% para el año 2006. La pregunta que surge es si estos docentes con una mayor cualificación académica están liderando la investigación en las universidades latinoamericanas o están pasando a ocupar los cargos administrativos en la institución.

Por otro lado, por lo menos en la legislación, se replanteo para la universidad una nueva concepción de investigación para crear un estamento de personal docente cualificado. Todos estos cambios inducían a preparar un profesional universitario para la docencia y la investigación. En definitiva, se da un cambio por lo menos en la legislación de las universidades latinoamericanas. Por una parte, a los docentes se les exigió investigación, pero por otra las políticas universitarias no lograron transformar las estructuras internas institucionales y se continúo con la función del docente universitario de "trasmisor de saberes". En este contexto nos encontramos que se impone un criterio financiero en la institución, al dar prioridad a la racionalización presupuestal donde la prioridad es el número de cursos que imparte el docente y se disminuye

\footnotetext{
${ }^{28}$ En el 2004 Colombia presentaba el 2\% de los programas de doctorado en Iberoamérica, ocupando el primer lugar Brasil con el $44 \%$ y seguido de España con el $23 \%$ y México con el $17 \%$.Se hace la relación sobre un total de 2.400 programas. ALDANA, Eduardo (2005): Doc. Cit., p.15.

${ }^{29}$ SNIES, Mayo del 2007.

${ }^{30}$ CNA.( abril, 2008): p. 4

31 En el Foro Internacional de Investigación. Investigación para la innovación, realizado en Bogotá, del 2 al 3 de junio del 2009, El Director del CAPES, de Brasil, Jorge ALMEIRA explicaba que graduaban once mil doctores por año y que ya estaban saturados en este nivel. Ahora, estaban impulsando los postdoctorados. Brasil presenta para el 20091.415 programas de doctorado y 2.696 de Maestría.

32 En la década de los noventa el $65,9 \%$ de los doctores se graduaron en las ciencias naturales (58) y el $28,4 \%$ lo hicieron en filosofía y teología (25). Las ingenierías, la agronomía, las ciencias sociales y las ciencias de la salud prácticamente no existían al nivel de doctorado. En los últimos ocho años se ha transformado dicha estructura.

33 En octubre del año 1998 ingreso la primera promoción del Doctorado en Ciencias de la Educación de RUDECOLOMBIA con 6 mujeres y 7 hombres. La primera graduada de esta promoción fue Miryam Báez Osorio, que de inmediato se vinculó de profesora del doctorado. Véase, Boletín de Historia de la Educación Latinoamericana, $N^{\circ} 12,2007$, p. 46.
} 
drásticamente los profesores de Planta ${ }^{34}$ dando prelación al profesor contratado que presenta un promedio de clases presenciales entre 16 a 24 horas semanales. ${ }^{35}$

En el tercer caso, de la expansión universitaria en América Latina, tema central en los años sesenta, se reitera la privatización de la universidad, en algunos países ${ }^{36}$ y se dan diferentes explicaciones a esta expansión universitaria que incluyen la feminización de la matrícula. ${ }^{37}$ En tal sentido, la mujer que había ascendido rápidamente en la matricula estudiantil llega a los albores del siglo XXI, en el 2002, "con una matrícula femenina total a nivel de pregrado en América Latina había superado el 54\%." ${ }^{38}$, especialmente en las carreras de educación y las humanísticas asociadas a las docencia. Se destaca Brasil con el $74,5 \%$, México con el $64,4 \%$ y Colombia con el $57 \%{ }^{39}$. Sin embargo, es generalizado el "techo de cristal" ${ }^{40}$ que no le deja ascender a la docencia universitaria sino en porcentajes de oscilan entre 20 y $30 \%{ }^{41}$ ni al espacio público negando "de esta manera la posibilidad alguna de ser reconocido, de ser oído y escuchado." ${ }^{.42}$

En todo caso, esta expansión de matrícula, planteó el problema de la formación y la capacitación del docente que requerían las universidades. La realidad es que "se

\footnotetext{
${ }^{34}$ Profesor de Planta hace referencia aquellos que ingresan por concurso y su contratación es indefinida.

${ }^{35}$ En Colombia, algunos docentes laboran en varias universidades. En el lenguaje coloquial se les denomina el profesor "bus" o el profesor "taxi" porque se bajan del bus o el taxi sólo para dictar el curso y de inmediato pasar a otra universidad.

${ }^{36}$ Por ejemplo, países como Argentina, Cuba, Venezuela la predominancia es de la pública.

37 "La expansión de las instituciones privadas y el crecimiento de la oferta de maestros han estado asociados a la feminización de la matrícula estudiantil y a la incorporación de nuevos estudiantes procedentes de hogares con menores ingresos económicos y de stock de capital humano", en RAMA, Claudio. (2006): pp. 14-15. Se indica de "recuperar la voz" que implica el rescate de sus saberes, conocimientos y perspectivas". MARTÍNEZ COVARRUBIAS, Sara. (2006): Mujeres y universidad. Vidas académicas. México, Universidad de Colima, p.23

${ }^{38}$ En el 2005 "casos como Uruguay, Venezuela y Cuba donde las tasas de matrícula son de 61, 60 y 75\% respectivamente". SIERRA, Rosaura y RODRIGUEZ, Gisela. (2005): Feminización de la matrícula de educación superior en América Latina y el Caribe. México, Unión de Universidades de América Latina, p.11. RAMA, Claudio (2006): Op. Cit, p. 15.

${ }^{39}$ POPADOPULOS Jorge y RADAKOVICH Rosario. (2005): Estudio comparado superior y género en América Latina y el Caribe. IESALC.

${ }^{40}$ Véase un amplio estudio sobre género en RUIZ TAGLE, Ana María (2008): Ni el aire que respiras. Pensamiento científico ante la violencia de género. Sevilla, Universidad Pablo Olavide de España, CAJASOL. Sobre las mujeres en la universidad en España y América Latina en FLECHA GARCIA, Consuelo y otras. (2008): Mujeres y universidad en España y América Latina. Buenos Aires, Niño y Dávila Editores. Sobre el ingreso de la mujer a la universidad véase ITATI PALERMO, Alicia. (2005):"El acceso de las mujeres a la universidad Argentina en el siglo XIX", en Mujeres y educación. Saberes prácticas y discursos en la historia, Buenos Aires, Argentina, pp. 413- 428.

${ }^{41}$ Véase las Tesis doctorales de Sol Mercedes Castro y Ana Hamon en el Doctorado Ciencias de la Educación de RUDECOLOMBIA.

${ }^{42}$ Se indica sin reconociendo no hay posibilidad de autocercioramiento, de identidad", en GUERRAPALMERO María José y DEL BASTO SABOGAL Liliana. (2009): Espacio público y perspectiva de género: en diálogo con el joven haberlas. Ibagué, Universidad del Tolima, pp. 115-116.
} 
pasó de 25.000 estudiantes universitarios en 1960 a 700.000 en 1994 sin que aumentara simultáneamente la capacidad de formación del docente y esta insuficiencia debilitó su labor pedagógica y la producción investigativa." ${ }^{43}$ En Colombia, se puede destacar que en un lapso de cinco años, de 1970 a 1975 , la matricula estudiantil aumentó de 80.000 a $176.000^{44}$ y en el Perú en 1993 el 85\% de la formación de docentes se realizaba en la Universidad Pública y en el 2002 bajo al 53\%. ${ }^{45}$

Finalizamos, el Siglo XX con reformas universitarias en la mayoría de los países de América Latina y del Caribe. A la universidad se le considera un bien público. Se retoma del Siglo XVIII el Control del Estado sobre la Universidad. Pero ahora se da un elemento nuevo como son los "estándares de evaluación y de calidad" que en Estados Unidos se venían aplicando desde finales del siglo XIX. ${ }^{46}$ Esta política trata de frenar lo laxo de la creación de las universidades privadas en varios países y por otra parte se incluye la evaluación y capacitación a su personal docente y ser más exigentes en la contratación de los mismos.

Asimismo, debemos indicar que iniciamos el siglo XXI con una crisis institucional de las universidades en América Latina unida a la crisis financiera que afecta a la universidad oficial. Y, no es un hecho aparte que continua en la palestra la función del profesor universitario en la institución. En especial, la formación que reciben de la institución a la que luego regresan motivados, quizá, por el factor pedagógico de formar y de enseñar o por no conseguir ubicación laboral en su profesión.

Por otra parte, un grave problema que está afectando a la universidad oficial es el de las jubilaciones de profesores de alta formación académica y en edad de productividad académica. Nos referimos a docentes que se jubilan a los cincuenta años y que se van como recurso humano a la universidad privada obteniendo estas instituciones docentes cualificados que no habían formado. Además, con una realidad de unos bajos salarios de los docentes en estas Universidades del Estado mientras las privadas de excelencia ofrecen una excelente prospectiva salarial.

En definitiva, sobre esta temática de la Universidad Latinoamericana, nosotros desde de Grupo de Investigación: «Historia y prospectiva de la Universidad Latinoamericana». HISULA, reconocemos la historia de la universidad dentro de su

\footnotetext{
43 BURBANO LÓPEZ, Galo. (1999): "La educación superior en la segunda mitad del siglo XX. Los alcances del cambio en América Latina y el Caribe", en Revista IBEROAMERICANA, de Educación. Madrid. OEI, p. 17

${ }^{44}$ CASTRO, Sol Mercedes. (2009): La mujer docente universitaria en Colombia. Formación e inserción laboral en la segunda mitad del siglo XX. Tesis doctoral. Doctorado Ciencias de la Educación, Universidad Pedagógica y Tecnológica de Colombia. UPTC., RUDECOLOMBIA, p. 192

${ }^{45}$ RAMA, Claudio. (2006): "La formación de docentes en América Latina: de las Normales a las universidades. ¿Solución o problema?, en SALGADO PEÑA Ramón Ulises (Compilador) (2006), Op. Cit., p. 14.

${ }^{46}$ Ibídem. pp. 15.
} 
contexto socio-político local, nacional e internacional como formadora del personal que dirige la sociedad en unos espacios de poder político y que estructura prácticas de culturas políticas que desde el siglo XVIII han incidido en la formación de las naciones latinoamericanas y ha intentado redefinir su misión y su participación en la sociedad.

Nos preguntamos sobre la relación de la universidad con la sociedad y su incidencia en el largo proceso de construcción de la nación ${ }^{47}$ desde las identidades ${ }^{48}$ regionales. ¿Cómo se han determinado los currículos ${ }^{49}$ planes y contenidos educativos, desde qué conceptos de nación y en qué relaciones de educación-estado-sociedad se han definido ellos, y cuál ha sido el efecto de esa determinación en la formación de la cultura política de los universitarios y su posterior práctica docente?

Por lo anterior, consideramos que "La universidad del siglo XXI es la comunidad académica de estudiantes y profesores reunidos en una institución para establecer un diálogo crítico teórico-práctico en un ambiente de libertad que promueve la esencia de su ser: la investigación científica interdisciplinar que genera «bienes públicos» que modifican los parámetros de la realidad establecida en un contexto cultural, sociopolítico y económico específico con visión internacional. $\rangle^{50}$ Por lo anterior, la misión de la universidad está dada desde la innovación ${ }^{51}$ para replantear la ciencia y la sociedad.

\footnotetext{
47 Tomamos en este trabajo la siguiente concepción de Nación "Es una comunidad política imaginada, construida, con forma histórica específica de organización social, y cohesión estatal como reflejo de la voluntad general", se ha tomado de tres autores: Anderson, Eder y Francis. Para Anderson: "Nación es una comunidad política imaginada como inherentemente limitada y soberana". ANDERSON, Benedict. (2007); Comunidades imaginadas. Reflexiones sobre el origen y la difusión del nacionalismo. México, Fondo de Cultura económica, p. 93. Para Eder: es algo construido, simbólico". EDER, Klaus (2003): Identidades colectivas e movilización de identidades, en Revista brasilera de Ciencias Sociales. Asociación Nacional de Pós - Graduação e Pesquisa em Ciências. São Paulo,p. 18. Para Francis “"Forma histórica específica de la organización social, que se da donde la mayoría de la población en un Estado moderno configura una unidad social, cuando dicha unidad se basa sobre todo en su cohesión estatal, y cuando el Estado es percibido como reflejo de la voluntad general". FRANCIS, Emerich (1957): Wissenschaftliche Grundlagen soziologischen Denkens. Muchen.

48 "Las identidades se construyen dentro, y no fuera, del discurso, tenemos que entender que están producidas a partir de estrategias enunciativas específicas, en ámbitos históricos institucionales específicos, en el seno de prácticas y formaciones discursivas específicas. Más aún, surgen dentro del juego de modalidades concretas de poder, de forma que son más el producto del señalamiento de la diferencia y la exclusión, que signos de una unidad idéntica naturalmente constituida". HALL, Stuart (1996) "Introduction: Who Needs Identity?" en S. Hall \& P. Du Gay (eds.): Questions of Cultural Identity, Londres, SAGE, p. 4.

49 Es interesante este estudio que se circunscribe en investigaciones del año 1981 y 1991 que replantean el currículo desde métodos de "problematización” Destacan ausencia de utopías, pensamiento neoconservador y se plantea un debate desde la sociología, retomar el pensamiento histórico y construir utopías. DE ALBA, Alicia (1997): El curriculum universitario de cara al nuevo milenio. México. UNAM, pp.10-23.

50 SOTO ARANGO, Diana. (2009): Los doctorados en Colombia, Op. Cit, p. 184.

51 Entendemos la innovación en las ciencias sociales como el "Conjunto de factores y actitudes que determinan que una empresa u organización del sector productivo o social genere, acepte e instrumente nuevas ideas, productos servicios y/o nuevos procesos que atiendan las necesidades cambiantes de la sociedad", véase en Relatoría "Formación para la investigación y la innovación”. Coordinadora: Dra. Wrana Panizzi. Brasil. Relatora: Dra. Diana Soto Arango. Colombia. Cartagena, 5 a 7 de octubre del 2005.
} 
La Visión de la Universidad latinoamericana en prospectiva hacia el año 2020 estará dada desde la inmersión político-social en la construcción de las naciones desde la interculturalidad y la educación interactiva de formación-capacitación". Reiteramos, que la propuesta de la "sociedad del saber" 52 es la más adecuada para los países de América Latina y en este camino necesariamente estará la Universidad en su calidad de responsable de formar el docente de esta institución.

\section{El profesor universitario en América Latina}

Consideramos que la pregunta clave para pensar ¿cómo se llega a ser profesor en una universidad latinoamericana? ¿Con qué valores éticos y competencias investigativas se le está formando para enseñar su disciplina? ¿Qué perfil presenta este profesional de la docencia universitaria? ¿Por qué al docente universitario no lo conceptualizamos como educador? ¿Por qué se ha perdido el título de maestro para este docente universitario?

Estas preguntas constituyen el marco de referencia sobre la conceptualización y por ende el perfil que se le otorga al profesor universitario. Asimismo, cabe preguntarse ¿qué implicaciones conceptuales se derivan en América Latina al utilizar la expresión de maestro sólo para el docente de primaria y a una persona que le haya dedicado toda su vida a la docencia con aportes significativos reconocidos en el ámbito académico? En efecto, lo que subyace en la respuesta es el imaginario de la persona que forma al niño en sus primeros años. ${ }^{53}$ En definitiva, si asociamos el término maestro a formar valores, conocimientos ¿por qué no se le otorga este título al profesional dedicado a la docencia en la Universidad?

Esta pregunta constituye una de las claves para pensar en el profesor universitario. En este sentido consideramos que existen dos tipos de docentes universitarios. Los primeros que se forman dentro de una disciplina y con la formación pedagógica que les orienta hacia la docencia. Nos referimos a los licenciados que van a desarrollar su trabajo en la básica primaria y secundaria pero que a su vez regresan, varios de estos con un grado adicional de postgrado, a la Facultad que los formó pero ahora en calidad de docentes. Precisamente, la bibliografía actual se orienta en este sentido, a los formados en las Facultades de Educación o en Ciencias Humanas.

\footnotetext{
52 UNESCO (1998): «Declaración sobre la Educación Superior en América Latina y el Caribe». En: Informe Final: Conferencia Mundial sobre la Educación Superior, UNESCO. -UNESCO (2009): "La Nueva Dinámica de la Educación Superior y la búsqueda del cambio social y el Desarrollo". CONFERENCIA MUNDIAL DE EDUCACIÓN SUPERIOR. París, Comunicado final, 05 - 08 de Julio de 2009.

53 En el Continente americano se le dedica un día de conmemoración al maestro. En América el 11 de septiembre se conmemora el Día Panamericano del Maestro, recordando el fallecimiento de Domingo Faustino Sarmiento, en el año 1888. En México y Colombia, se celebra el Día del Maestro' el 15 de mayo, en Uruguay el 22 de septiembre, Perú se celebra el 6 de julio de cada año en todo el país, en Chile se celebra el 16 de octubre, conmemorando la fundación del Colegio de Profesores del país. En España se celebra el Día del Maestro en honor de José de Calasanz el 27 de noviembre. Fuente. Wiquipedia.
} 
El segundo docente universitario, nos referimos aquel profesional que sólo recibió formación en su disciplina científica y que por diferentes motivos personales regresa a la universidad en calidad de docente. Para este profesional se ha considerado que con unas "técnicas o herramientas" didácticas puede desarrollar de manera excelente su trabajo docente.

Efectivamente, a este profesional universitario, que ha sido capacitado en una disciplina científica, cabe la pregunta si se le habilitó "para generar y gestionar nuevos productos y servicios a la sociedad" o ¿simplemente se le adiestró en una disciplina? ${ }^{54}$ Es decir, se le preparó para ser innovador en su profesión o sólo fue un entrenamiento profesional? Es más, en el perfil de las profesiones que no estén relacionadas con la docencia no se incluye la función educadora. Y, en este último plano estamos entrando en la idoneidad de ciudadanos que con lleva la formación en valores de ética y sociales.

Por lo tanto, el primer eje de análisis sobre el profesor universitario, se debe centrar en reconocer unas personas que habilita la universidad, dentro de una ética-científico de su disciplina y como docente que corresponde a un actor socio-político que está configurado en grupos y redes de poder. El reto es: interrelacionar la capacitación disciplinar-científica con la ética del educador-científico en el sistema educativo universitario.

En el estricto sentido el profesor universitario tendrá que conocer muy bien su disciplina y especialmente le corresponde estar investigando sobre la misma. La tendencia de futuro es que la docencia que realice se vincule a la investigación porque es allí, donde la docencia-investigación interrelaciona los valores éticos y se interactúa con las personas que forma, construyendo conocimiento dentro de la tolerancia y la inclusión.

Cabe anotar que si bien este docente se localiza, como hemos indicado, en una institución y en un contexto socio-político, en la práctica la normativa institucional es la que le permite en mayor o menor grado desarrollar ese "maestro" que le motivo optar profesionalmente por ser profesor universitario.

No pretendemos desconocer que este docente que realiza su ejercicio profesional en la universidad tiene una historia de vida. Estas se han analizado en estudios del Instituto Internacional para la Educación Superior en América Latina y el Caribe. IESAL. Este es el caso de los análisis del origen socio-económico de los docentes que llegan a las Facultades de Educación y Humanidades, donde se establece que provienen de estratos de menores ingresos socio-económicos ${ }^{55}$ y que estas carreras requieren de menores puntajes de ingreso. Pero esta visión, la podemos ubicar en los inicios de la

\footnotetext{
54 BANCO MUNDIAL. (1998): Recomendaciones del Banco Mundial para la Educación Superior expuestas en la UNESCO, http://contextoeducativo.com.ar/1999/12/Maglio/ bancomundial.htm

${ }^{55}$ Consideran que "las capas medias o altas prefieren incentivar a sus hijos a que seleccionen las orientaciones educativas que están asociadas a salarios profesionales más altos". SALGADO PEÑA Ramón Ulises () (2006), p. 17.
} 
formación del nuevo docente. Sin embargo, el docente universitario que actualmente llega a la universidad proviene desde las diferentes profesiones y cada vez con un nivel de cualificación más alto por exigencia de las instituciones. En tal sentido, son relevantes los estudios particulares de algunas universidades sobre la función del docente universitario ${ }^{56}$ y que contribuyen a dar un marco de referencia sobre las motivaciones y problemáticas de este profesional en la universidad. ${ }^{57}$

Resulta interesante que para América Latina, los Proyectos Tunning y $6 \mathrm{X} 4,{ }^{58}$ se preocupen por establecer perfiles para el docente universitario. De esta manera en el Proyecto 6X4 se establecía las siguientes competencias Genéricas, entendiendo ésta como: "la capacidad efectiva para realizar una actividad o tarea profesional determinada y los criterios de ejecución y evidencia de realización." 59 De esta manera, el profesor universitario debería habilitarse para la docencia; la investigación; poseer los conocimientos de la disciplina; poseer métodos, técnicas y capacidad de análisis y síntesis para procesar la información; desarrollar capacidad creativa para la innovación; capacidad para orientar la investigación a soluciones sociales; capacidad para organizar los conocimientos independientes para la solución de problemas. En el estricto sentido, se refiere a poseer actitudes, habilidades y conocimientos para la investigación y la innovación.

Por lo que hemos podido analizar se localizan investigaciones que muestran los paradigmas del ejercicio docente que se remontan al tradicional; humanista; tecnológico; indagativo. Igualmente, es necesario destacar las publicaciones que analizan las tendencias "latinoamericanas emergentes" donde aparece la "del desarrollo humano integral, de una nueva solidaridad y cooperación internacional." ${ }^{\prime 60}$ De forma paralela, se aboga por la formación del docente dentro de la interculturalidad como un reto socio-educativo y profesional en el sigloXXI. ${ }^{61}$ Sin duda, otro indicador común en los estudios es el de incluir la investigación y de la necesidad de la capacitación permanente del docente universitario.

\footnotetext{
56 Véase un estudio sobre el profesor universitario en la Universidad Distrital "Francisco José de Caldas" de Bogotá, Colombia. ORTIZ MOLINA Blanca Inés; GARCIA SANCHEZ Barbará; SANTANA GAITAN, Luis Carlota. (2008): El trabajo académico del profesor universitario. Bogotá, la Universidad Distrital "Francisco José de Caldas.

57 Véase documento citado ORTIZ MOLINA Blanca Inés; GARCIA SANCHEZ Barbará, SANTANA GAITAN, Luis Carlota. (2008).

58 El Proyecto 6X4 UELAC. Un diálogo universitario", se inició en la ciudad de Guadalajara, México el 14 de abril del 2005, bajo la dirección del CENEVAL. En El 2006 finalizó con la reunión en la Universidad de Mendoza, Argentina, bajo la coordinación de ASCUN. Véase un análisis en SOTO ARANGO, Diana. (2007): "La investigación y la innovación en los programas de historia y Ciencias sociales en Colombia. Una propuesta desde el proyecto 6X4", en Revista Historia de la Educación Latinoamericana, Número 9, pp.203-230.

59 VERDEJO, Pilar. (2006): "Reunión de ciudad de México del Proyecto 6X4", México, abril. ${ }^{60}$ MIRANDA Christian. (2003): "la formación del docente en Chile. Investigación tendencias y práctica", en Formación del profesorado en América Latina: diversos contextos socio-políticos. Bogotá, Ediciones Antropos, pp. 71-72.

${ }^{61}$ La interculturalidad la entienden como "un principio y un proyecto para la mejora de acciones y relaciones entre las culturas, configurada mediante el diálogo y el encuentro compartido entre todos los estudiantes, el profesorado y las comunidades participantes". DOMINGUEZ GARRIDO, María Concepción. (2006): Investigación y formación del profesorado en una sociedad intercultural. Madrid, Editorial universitaria, S.A, pp. 12.
} 
Pero el tema central es cómo se da la solución a los principales problemas actuales que afectan la formación y el ejercicio del docente universitario. En primera instancia, el de la formación, parece no ser relevante al existir el imaginario que un docente puede ser cualquier profesional. Lo anterior, va unido a la parte legislativa en algunos países. En este contexto se inscribe Colombia, donde ya no se requiere formación pedagógica para enseñar en los niveles de básica primaria y secundaria. En la universidad, las condiciones de ingreso para un docente se centran en el examen del perfil disciplinario pero en pocos países se solicita, en los concursos de ingreso, el proyecto docente que demuestre la capacitación pedagógica.

En segunda instancia, se indica que la preocupación institucional en el ejercicio profesional del docente universitario se orienta fundamentalmente al hecho de dictar la cátedra. En efecto, se correlaciona presupuesto con el número de horas que imparte el profesor. De esta manera, encontramos un promedio de 16 horas en los profesores de planta y de 24 en los profesores contratados. Es decir, al docente se le establece como actividad prioritaria el hecho de "impartir clases". Por otra parte, dentro del contexto del profesor "dictador de clases" unido a la baja remuneración y a la "minusvaloración" 62 de su trabajo se convierte en el docente "bus" o "taxi", término utilizado para identificar a los profesores universitarios que ejercen la docencia en varias instituciones. Por ello es necesario seguir insistiendo en los estatutos del profesor investigador que permitan el ejercicio de una docencia digna con calidad investigativa y que la evaluación docente se realice sobre su producción académica e igualmente sobre ese imaginario que deja en los estudiantes respecto a la ética, la tolerancia, el servicio ${ }^{63}$ y la inclusión".

Pero volviendo a nuestro tema central de argumentación, consideramos que el profesor universitario debe ser formado para investigar y para enseñar a investigar. Es decir la función docente es investigativa. Por ello, es necesario seguir insistiendo en que el perfil del profesor universitario está acompañado de la capacidad para desarrollar innovación e investigación. Pero debemos resaltar que en Ciencias Sociales la innovación también genera "bienes públicos como las ideas, conceptos y modifica parámetros de la realidad." ${ }^{64}$ En definitiva, el docente universitario, desde la libertad de pensamiento, repensará la sociedad y replanteará conceptos que modifiquen cambios de conductas siempre dentro de los valores éticos ciudadanos de la comunidad de América Latina.

Por otra parte, es erróneo considerar que la capacidad de innovar es una característica exclusiva del ámbito del postgrado. En tal sentido, es prioritario vincular en la docencia, desde los primeros años del pregrado, el elemento integrador de las líneas de investigación en los grupos de investigación interdisciplinarios.

\footnotetext{
${ }^{62}$ VASCO, Carlos. (2005): "Pedagogías para la comprensión en las disciplinas académicas", en Retos de la universidad contemporánea. Bogotá, Universidad Distrital «Francisco José de Caldas», p. 59.

${ }^{63}$ Esa capacidad que tiene el maestro de vincularse en la solución del problema académicopersonal del su estudiante. El servicio a los estudiantes se da con el ejemplo de vida, con la generosidad que practique el profesor y que luego el nuevo profesional lo replicará en las nuevas generaciones.

${ }^{64}$ Relatoría Diana Soto Arango, Proyecto 6X4. Cartagena, Oct. 2005.
} 
No desconocemos que América Latina, está fuera de los indicadores relacionados con la innovación según la clasificación de las universidades en el mundo que nos deja en últimos lugares al medir los premios Nobel y artículos científicos en circulación. ${ }^{65}$ No deja de ser irónico que en Colombia el único premio Nobel Gabriel García Márquez, no fue a la universidad y sin embargo es uno de los grandes pensadores para latinoamérica no sólo en literatura sino en educación. ${ }^{66}$

Reiteramos, que es especialmente importante para el desarrollo de los docentes universitarios la cooperación entre los países europeos y latinoamericanos, hecho que ya se observa en el incremento del número de Redes de investigación. ${ }^{67} \mathrm{~A}$ través de diversas reuniones y organizaciones ${ }^{68}$ se han perfilando acuerdos sobre cómo instrumentar y hacer realidad los propósitos de ambos espacios. ${ }^{69}$ Igualmente, no se puede desconocer que el problema de la investigación en la universidad es prioridad del momento en reuniones de Ministros, ${ }^{70}$ Rectores y Académicos que piensan en el futuro de la universidad.

En definitiva, el desarrollo de la capacidad para la innovación en la formación de los investigadores y profesionales es un elemento necesario para el intercambio y transferencia de conocimientos, y para el fomento de capacidades emprendedoras.

En este contexto, el perfil del profesor universitario puede concebirse ${ }^{71}$ desde:

\footnotetext{
65 Marzo 21 del 2008. Según el último escalafón de las 500 mejores universidades del mundo, que anualmente realiza la Universidad Jiao Tong, de Shangai (China), no hay ninguna universidad colombiana en este ranking, que está encabezado por la Universidad de Harvard y en el que la primera institución de Suramérica es la Universidad de Sao Paulo -Brasil-, en el puesto 141 y la Universidad de Buenos Aires -Argentina-, en el puesto 172.

66 VASCO, Carlos Eduardo y otros. (1996): Misión de los sabios. Colombia: al filo de la oportunidad, Bogotá, 21 de julio de 1994. TERCER MUNDO EDITORES.

67 SEBASTIÁN, Jesus. (1999). Análisis de las redes de investigación de América Latina con la Unión Europea. RECITEC, Recife, v. 3, n. 2, p. 308-321.

${ }^{68}$ COOKE, P. (2004): University Research and Regional Development. A Report to ECDG Research . Documento de investigación. European Commission. En http://europa.eu.int/comm/ research/conferences/2004/univ/pdf/univ_regional;_MINISTRY of Science Technology and Innovation. (2005, February). A Framework for Qualifications of The European Higher Education Area [Versión electrónica] Bologna Working Group on Qualifications Frameworks. , en http://www.bologna-bergen 2005.

${ }^{69}$ VI Taller de indicadores de ciencia y tecnología - Interamericano e Iberoamericano. (2004, 15 al 17 de septiembre)-Buenos Aires. Red de Indicadores de Ciencia y Tecnología (RICYT) Iberoamericana / Interamericana CYTED OEA Programa Iberoamericano de Organización de Ciencia y Tecnología para el Desarrollo Estados Americanos.

70 CUMBRES IBEROAMERICANAS. Análisis cumbres iberoamericanas y jefes de Estado, En www.cumbresiberoamericanas.com/; -COMUNIDAD ANDINA (2004). Reuniòn del Consejo de Ministros y responsables de Políticas culturales de la Comunidad Andina. Quito República del Ecuador, 10 de julio del 2004. CONFERENCIA Científica de la V Cumbre Iberoamericana de Jefes de Estado y de Gobierno. (1995, octubre). Formación para la Innovación. Buenos Aires, Argentina. - REUNIÓN DE MINISTROS (2005): ANEXO II. Declaración de la II Reunión de Ministros de Educación de América Latina y el Caribe y la Unión Europea. Ciudad de México, 14 y 15 de abril del 2005.

${ }_{71}$ Síntesis de los planteamientos del Proyecto 6X4.
} 
1. Aplicación del conocimiento para generar y gestionar nuevos productos. Categorías conceptuales

2. Diseño y desarrollo de proyectos de investigación en el contexto de la profesión con la calidad y actualidad académica adecuada.

3. Dominio de los aspectos teóricos y metodológicos de la investigación en pedagogía y didáctica.

4. Creación y gestión de organizaciones para la investigación y la innovación. Nos referimos a que sean líderes de grupos de investigación. Es decir, que desarrollen actitudes de liderazgo, colaboración, crítica, innovación e investigación y emprendimiento en la profesión.

6. Desarrollo de proyectos interdisciplinares y transdiciplinares (crear nuevas disciplinas)

7. La aplicación de criterios y valores éticos personales y para la profesión que respondan a las necesidades institucionales, regionales, nacionales y de Iberoamérica.

En todo caso, por lo que hemos podido analizar no se puede concebir ni investigar la función del profesor universitario sino esta unido a la reflexión seria, rigurosa y profunda sobre la historia y prospectiva de la universidad. ${ }^{72}$ Especialmente, sobre el papel que ha jugado esta institución en la formación de profesionales que luego se vincularán al ejercicio de la profesión, la docencia, la política. Es decir, la universidad formando a los dirigentes del país. En definitiva, es hacer inclusiva ${ }^{73}$ la Universidad y que la función docente sea "educativa en tanto es una función cultural que confiamos el mundo a nuevos sujetos." 74

\section{CONCLUSIONES}

En este trabajo partimos de la pregunta ¿si la universidad en América Latina ha formado su cuerpo docente con capacidad profesional de liderar la transformación cultural-educativa, y de liderar los cambios científicos-tecnológicos-sociales desde la ética, la solidaridad, la tolerancia, la inclusión en las "sociedades del saber" cambiante del siglo XXI.

Hemos localizado, en primera instancia, que la universidad ha representado la historia de una tensión ideológica que involucra visiones sobre el mundo y las sociedades. Sin duda, es el espacio donde se puede pensar libremente porque esta es la razón de ser de esta institución y en el estricto sentido, la universidad ha formado, generalmente, a quienes luego se vinculan como docentes de la misma. Sin embargo, la universidad latinoamericana ha estado alejada de la modernidad y de los standares de investigación internacional. La

\footnotetext{
72 En el siglo XVIII se establece claramente la diferencia entre las denominadas Universidades Menores y Mayores. Las Universidades MENORES o PARTICULARES correspondió aquellas instituciones universitarias con cátedras y privilegios limitados, con facultades restringidas para graduar, universidades caseras , de categoría inferior .. sobre las cuales se legisla de un modo concreto

73 Una educación para todos y todas sin discriminación de raza, nivel socio-económico, cultural, tendencia .sexual, pensamiento político.

74 MANJON RUIZ, Juana. (2000): "Algunas funciones del profesor universitario para el siglo XXI. Consideraciones éticas", en Revista FUENTES, Vol. 2. Universidad de Sevilla, Facultad Ciencias de la Educación, p. 67.
} 
universidad fue pensada, principalmente, en el siglo XX, por los estudiantes y afecto la institución la masificación y el ingreso de la mujer. A finales del siglo XX se dieron una serie de reformas universitarias que han incidido en la evaluación de la universidad y en la formación del profesional que regresa de docente a la institución.

Respecto a la modernidad nos preguntamos si ¿La universidad Latinoamericana y del Caribe ha evolucionado para asumir la modernidad?:Qué estrategias coadyuvan a consolidar en la modernidad la universidad Latinoamericana para cristalizar la transformación de la tercera revolución industrial?; ¿Cuáles son las culturas, tradiciones, desarrollos, obstáculos e inercias, desde la Universidad Latinoamericana y del Caribe para abordar la problemática de nación y modernidad?; y ¿Cuál es la cultura de cambio que se vive en la universidad de América Latina y el Caribe? ¿Cómo se concibe la universidad en 20 años?

Por otra parte, en el estudio especifico de la formación del docente universitario en Latinoamérica nos preguntamos de ¿cómo se llega a ser profesor universitario? y ¿cómo actúa sobre las personas que forma?. Se ha localizado, como es lógico, que es diferente la formación del graduado en las Facultades de Educación al de las otras facultades. Sin embargo, este profesor universitario independiente de la Facultad que provenga es deficiente en las investigaciones y publicaciones internacionales que son medidas por el ranking universitario.

Se encuentra grandes falencias en el profesor universitario producto quizá de la formación pero esencialmente por las estructuras académicos legales de la universidad que da prioridad al profesor "dictador de clases". La investigación junto con la innovación que se oriente a la creación de ideas, conceptos que modifiquen los parámetros de la realidad generando "bienes públicos" que incidan en su medio socio-cultural, es aún un largo camino por recorrer en las universidades latinoamericanas.

En efecto, este es el gran desafío de la universidad habilitar profesores universitarios desde la diversidad, la interculturalidad. En este sentido, las políticas de desarrollo de las instituciones universitarias se deben orientar hacia la preparación de un docente que comprenda su entorno y su historia, preparándolo de esta manera para el ingreso de las sociedades del saber. El gran reto que se nos aproxima es el adecuado manejo del ciberespacio de la universidad virtual. El desarrollo de las metodologías como la disciplinar y transdiciplinar ¿cómo se van a desarrollar en las investigaciones?. Pero más que la tecnología lo que se enfrenta la universidad en el siglo XXI es a una disminución radical de la población que regularmente se encontraba en la universidad en edades de 17 a 25 años. La población mundial envejece $\mathrm{e}^{75}$ y por lo tanto, los profesores deben ser formados para atender igualmente a este sector de la población.

75 CEPAL (2009). Observatorio demográfico. - NACIONES UNIDAS (1998): Fondo de población de las Naciones Unidas (FNUAP): Estado de la Población Mundial 1998. IMD World Competitiveness Yearbook. World Economic Forum: OCDE. Red de Indicadores de Ciencia y Tecnología (RICYT). (2004, septiembre). VI Taller de indicadores de Ciencia y Tecnología - Interamericano e Iberoamericano. Buenos Aires, 15 - 17 de septiembre de 2004. ONU (2006); Informe perspectiva de la población mundial, revisión de 2006. www.un.org/esa/policy/ 
Por todo lo anterior, consideramos que el profesor universitario requiere de una redefinición conceptual, legal de su práctica docente dentro de los criterios de la nueva visión de la universidad. Por otra parte, el docente universitario, al tener bajo su responsabilidad la formación de la élite dirigente del país y de quienes ocuparan su lugar en años posteriores, en la docencia universitaria, debe clarificar que la formación del nuevo ciudadano se realiza desde el ejemplo de su ejercicio profesional. Valores y actitudes como: la tolerancia, respeto a los derechos humanos y a la interculturalidad, la diversidad, la equidad de género, la innovación, el liderazgo desde la identidad a la institución, se orientarán a la construcción de nación. Esta relación de estudiante - profesor será la determinante para la identidad y el futuro del nuevo profesional. Relación que se da no precisamente en el aula de clase sino en el proceso investigativo que realice con los estudiantes en el curso, actividades de extensión y direcciones de tesis. Cabe preguntarse en este marco ¿Quién no recuerda a ese profesor que demostró el "don de servicio" y generosidad por el estudiante?

De forma paralela, la responsabilidad que tiene el profesor universitario, de conocer la disciplina que enseña con los últimos avances en la misma que se debe demostrar a través de las redes y grupos de investigación, nacional e internacionales, a los cuales está vinculado. Asimismo, el conocimiento didáctico unido al proceso investigativo para que el estudiante asuma los nuevas prácticas educativas dentro de los grupos de investigación. Efectivamente, no se puede concebir en el siglo XXI un profesor y un estudiante universitario que no se encuentre vinculado a un grupo de investigación. Es allí donde construye los imaginarios de identidad institucional y profesional.

Pero encontramos también que la formación futuro profesor universitario, precisamente, el que va a liderar la universidad, en escasos diez años, se debe responder a las preguntas sobre: ¿A qué problemas prioritarios deben reconocer las ofertas de formación en nuestras universidades?; ¿Cuáles son las cotidianidades que hacen visible una cultura con unas prácticas científico-éticas en relación con el conocimiento en las universidades de cada país y región?; ¿Qué aportes, retos y desarrollos han sugerido las nuevas leyes, decretos y resoluciones para la Educación Superior en relación al desarrollo de la ciencia y Tecnología? ¿Cuál ha sido su impacto en los Currículos Universitarios? ¿Están nuestras universidades y docentes preparados para el cambio conceptual de la universidad?

En definitiva, estas preguntas constituyen las claves de la institución a tener en cuenta en la formación del docente universitario que por otro lado está unido al reto del profesor de lograr la transformación de la universidad pero reiteramos desde los valores de solidaridad y cooperación frente a la sociedad de la individualidad y la falta de tolerancia porque primero se es persona y luego profesional.

\section{FUENTES}

Banco Mundial (1998): Recomendaciones del Banco Mundial para la Educación Superior expuestas en la UNESCO, 1998.http://contextoeducativo.com.ar/1999/12/Maglio/ bancomundial.htm.

CEPAL. (2009). Observatorio demográfico. 
Diana Soto Arango

CUMBRES IBEROAMERICANAS. Análisis cumbres iberoamericanas y jefes de Estado, en www.cumbresiberoamericanas.com.

CNA. (Abril, 2008): Situación Actual de los Doctorados en Colombia: Análisis de Indicadores que tipifican características importantes. Bogotá. Documento de Trabajo.

COMMISSION of the European Communities (2003, July). Communication from the Commission to the Council and the European Parliament. Researchers in the European Research Area: one profession, multiple careers [Version electrónica], Brussels: COM., en http://europa.eu.int/comm/research/fp6/mariecurie; actions/pdf/careercommunication.

COMUNIDAD ANDINA. (2004): Reunión del Consejo de Ministros y responsables de Políticas culturales de la Comunidad Andina. Quito República del Ecuador, 10 de julio del 2004.

CONFERENCIA Científica de la V Cumbre Iberoamericana de Jefes de Estado y de Gobierno. (1995, Octubre), Formación para la Innovación. Buenos Aires, Argentina.

COOKE, P. (2004): University Research and Regional Development. A Report to EC-DG Research. Documento de investigación. European Commission. En http://europa.eu.int/ comm/research/conferences/2004/univ/pdf/univ_regional.

MINISTRY of SCIENCE TECHNOLOGY AND INNOVATION. (2005, February): A Framework for Qualifications of The European Higher Education Area [Versión electrónica] Bologna Working Group on Qualifications Frameworks, en http:// www.bologna-bergen 2005.

NACIONES UNIDAS. (1998): Fondo de población de las Naciones Unidas (FNUAP) : Estado de la Población Mundial 1998. IMD World Competitiveness Yearbook. World Economic Forum: OCDE. Red de Indicadores de Ciencia y Tecnología (RICYT). (2004, septiembre). VI Taller de indicadores de Ciencia y Tecnología - Interamericano e Iberoamericano. Buenos Aires, 15 - 17 de septiembre de 2004. ONU (2006); Informe perspectiva de la población mundial, revisión de 2006. www.un.org/esa/policy.

MINISTRY of Science Technology and Innovation. (2005, February): A Framework for Qualifications of The European Higher Education Area [Versión electrónica] Bologna Working Group on Qualifications Frameworks. , en http://www.bologna-bergen2005.

MORENO Y ESCANDON, Francisco Antonio. (1774): "Método provisional e interino de los estudios que han de observar los colegios de Santafé por ahora, y hasta tanto se erija Universidad Pública, o su Majestad dispone otra cosa", Santafé, septiembre 22 de 1774 , en Archivo General de Indias. Sección Quinta, Audiencia de Santa Fe, Legajo 759.

NACIONES UNIDAS. (1998): Fondo de población de las Naciones Unidas (FNUAP): Estado de la Población Mundial.

IMD World Competitiveness Yearbook. World Economic Forum: OCDE. Red de Indicadores de Ciencia y Tecnología (RICYT). (2004, Septiembre). VI Taller de indicadores de 
Ciencia y Tecnología - Interamericano e Iberoamericano. Buenos Aires, 15 - 17 de septiembre.

ONU. (2006): Informe perspectiva de la población mundial, revisión de 2006. www.un.org/ esa/policy/.

REUNIÓN DE MINISTROS. (2005): ANEXO II Declaración de la II Reunión de Ministros de Educación de América Latina y el Caribe y la Unión Europea. Ciudad de México, 14 y 15 de abril.

(RICYT) VI Taller de indicadores de ciencia y tecnología - Interamericano e Iberoamericano. (2004, 15 al 17 de septiembre) Buenos Aires. Red de Indicadores de Ciencia y Tecnología (RICYT) Iberoamericana / Interamericana CYTED OEA Programa Iberoamericano de Organización de Ciencia y Tecnología para el Desarrollo Estados Americanos.

SNIES, a febrero de 2009. Observatorio de la Universidad Colombiana.

UNESCO (1998): Conferencia Mundial sobre Educación Superior para el siglo XXI. París del 5 al 9 de octubre de 1998. Publicado en UNESCO (1998): Informe Mundial sobre la Educación, Ed. Santillana, UNESCO, Madrid, España. Y el "Informe Mundial sobre la Ciencia" (1998): Madrid, Ed. Santillana, UNESCO.

UNESCO. (1998): Recomendaciones del Banco Mundial para la Educación Superior expuestas en la UNESCO, 1998. http://contexto-educativo.com.ar/1999/12/Maglio/ banco mundial.

UNESCO. (1998): "Declaración sobre la Educación Superior en América Latina y el Caribe", en Informe Final: Conferencia Mundial sobre la Educación Superior.

UNESCO. (2009): “La Nueva Dinámica de la Educación Superior y la búsqueda del cambio social y el Desarrollo". CONFERENCIA MUNDIAL DE EDUCACIÓN SUPERIOR, París, Comunicado final, 05 - 08 de Julio de 20.

VALENZUELA, Eloy. (1806): Plan que ha de servir de método para el estudio de la filosofia en el Colegio de San Pedro Apóstol de la Villa de Mompox. : Biblioteca Nacional de Colombia. Sala Raros y Curiosos, Sección Manuscritos, No. 358, folios 298 a 309.

VASCO, Carlos Eduardo y otros. (1996): Misión de los sabios. Colombia: al filo de la oportunidad, Bogotá, 21 de julio de 1994, TERCER MUNDO EDITORES.

VERDEJO, Pilar, “Reunión de ciudad de México del Proyecto 6X4,” México abril, 2006.

\section{SELECCIÓN BIBLIOGRÁFICA}

ALDANA, Eduardo. (2005): “Misión de los sabios. Colombia: al filo de la oportunidad", Bogotá, Tercer Mundo Editores.

BAEZ OSORIO, Miryam. (2006): Las Escuelas Normales. Época radical. Tunja, Colección 
Tesis doctorales, Doctorado Ciencias de la Educación, RUDECOLOMBIA, Universidad Pedagógica y Tecnológica de Colombia.

BURBANO LÓPEZ, Galo. (1999): "La educación superior en la segunda mitad del siglo $\mathrm{XX}$. Los alcances del cambio en América Latina y el Caribe", en Revista Iberoamericana de Educación, Madrid, OEI.

CASTRO, Sol Mercedes. (2009): "La mujer docente universitaria en Colombia. Formación e inserción laboral en la segunda mitad del siglo XX”, Tesis doctoral, Doctorado Ciencias de la Educación, Universidad Pedagógica y Tecnológica de Colombia. UPTC, RUDECOLOMBIA.

DOMINGUEZ GARRIDO, María Concepción. (2006): Investigación y formación del profesorado en una sociedad intercultural, Madrid, Editorial universitaria, S.A.

MANJON RUIZ, Juana. (2000): “Algunas funciones del profesor universitario para el siglo XXI. Consideraciones éticas", en Revista FUENTES, Vol. 2, Universidad de Sevilla, Facultad Ciencias de la Educación.

MIRANDA Christian. (2003): "la formación del docente en Chile. Investigación tendencias y práctica", en Formación del profesorado en América Latina, diversos contextos sociopolíticos, Bogotá, Ediciones Antropos.

MÚNERA, Alfonso (1998): El fracaso de la nación. Región clase y raza en el Caribe colombiano. 1717-1810, Bogotá, Banco de la República. El Áncora Editores.

ORTIZ MOLINA, Blanca Inés, GARCIA SANCHEZ, Barbará, SANTANA GAITAN, Luis Carlota. (2008): El trabajo académico del profesor universitario. Bogotá, Universidad Distrital Francisco José de Caldas.

POPADOPULOS, Jorge y RADAKOVICH, Rosario. (2005): Estudio comparado superior y género en América Latina y el Caribe. IESALC.

RODRIGUEZ CRUZ, Agueda. (1990): Historia de la universidad de Salamanca, Salamanca, Fundación Ramon Areces.

SALGADO PEÑA, Ramón Ulises. (2006): "La formación docente en la región: de las normales a las universidades", en Informe sobre la educación superior en América Latina y el Caribe 2000-2005, Caracas, Instituto Internacional de la UNESCO para la Educación Superior de América Latina y el Caribe.

SALGADO PEÑA, Ramón Ulises. (2006): La formación de docentes en América Latina, Tegucigalpa, Fondo Editorial Universidad Pedagógica Nacional Francisco Morazán.

SEBASTIAN, Jesus. (1999): Análisis de las redes de investigación de América Latina con la Unión Europea, RECITEC, Recife, v. 3, n. 2.

SOCARRÁS, José Francisco. (1987): Facultad de Educación y Escuela Normal Superior. 
Su historia y aporte cientifico, humanistico y educativo, Tunja, Ediciones la Rana y el Águila.

SOTO ARANGO, Diana. (1993): Polémicas universitarias en Santa Fe de Bogotá. Siglo XVIII, Bogotá, Universidad Pedagógica Nacional - COLCIENCIAS.

. (2003): El modelo de reforma universitaria Ilustrada en la metrópoli y colonias americanas, Córdoba, Universidad de Córdoba.

(2004): La reforma del Plan de estudios del Fiscal Moreno y Escandón. 1774-1779, Bogotá, Universidad Colegio Mayor del Rosario.

. (2007): "La investigación y la innovación en los programas de historia y Ciencias sociales en Colombia. Una propuesta desde el proyecto 6X4", en Revista Historia de la Educación Latinoamericana, No 9 Tunja, Universidad Pedagógica y Tecnológica de Colombia, RUDECOLOMBIA, Sociedad de Historia de la Educación Latinoamericana.

. (2007): La autonomía en la universidad colombiana. Nuevo Reyno de Granada, en Autonomía y modelos universitarios en América Latina. SOTO ARANGO, Diana y LAFUENTE GUANTES, María Isabel. Tomo II Colección Historia y prospectiva de la universidad latinoamericana, Universidad de León, España, Asociación Colombiana de Universidades, ASCUN, Universidad Pedagógica y Tecnológica de Colombia, RUDECOLOMBIA.

. (2009): "Los doctorados en Colombia. Un camino hacia la transformación universitaria", en Revista Historia de la Educación Latinoamericana, No 12, Tunja, Universidad Pedagógica y Tecnológica de Colombia, RUDECOLOMBIA, Sociedad de Historia de la Educación Latinoamericana.

VALENCIA, Carlos Hernando. (2006): Las Escuelas Normales y la formación del magisterio. Primera mitad del siglo XX, Manizales, Colección Tesis doctorales, Doctorado Ciencias de la Educación, RUDECOLOMBIA, Universidad de Caldas.

VASCO, Carlos. (2005): "Pedagogías para la comprensión en las disciplinas académicas", en Retos de la universidad contemporánea, Bogotá, Universidad Distrital Francisco José de Caldas.

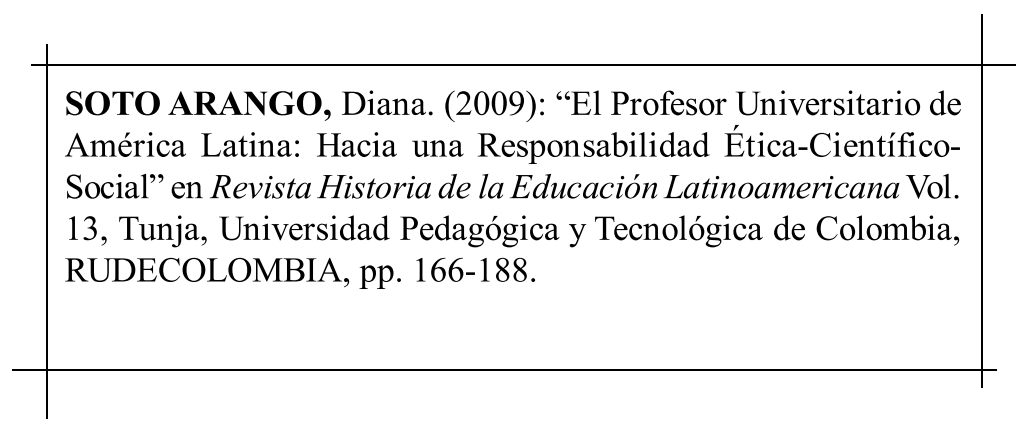

Rhela. Vol. 13. Año 2009, pp. $166-188$ 